\title{
EDITORIAL
}

\section{The current status of minimal residual disease assessment in myeloma}

Leukemia (2014) 28, 239-240; doi:10.1038/leu.2013.306

By most definitions of cure, myeloma remains an incurable disease. 'Functional cure' denoting long-term disease control has been described in the context of intense therapies, and it is quite likely that a small fraction of these patients have a complete eradication of the myeloma clone with no more than average risk of myeloma recurring ('real cure'). ${ }^{1-3}$ However, we have been hampered by the lack of a sensitive way to determine the presence of residual tumor cells as has been possible with chronic myelogenous leukemia, where the universal presence of $\mathrm{Bcr}-\mathrm{Abl}$ translocation provides the ability to detect extremely small numbers of tumor cells. ${ }^{4}$ For the longest time, the need for a sensitive method to determine small amounts of residual tumor was not perceived, primarily because of the inability to achieve even the currently defined deepest remission state (complete response, $\mathrm{CR}$ ) in any substantial number of patients with available treatments. However, with newer treatment approaches, we can achieve $C R$ in a large majority of patients, including a significant proportion with stringent $C R$ representing lack of clonal plasma cells in the marrow by immunophenotyping. ${ }^{5-7}$ Despite this level of response, studies have shown that the vast majority of patients have residual tumor cells that can be detected with a variety of sensitive techniques. ${ }^{8-11}$ This in turn brings up three important questions: (a) what are the implications of obtaining a minimal residual disease (MRD) negative status in myeloma, (b) what is the ideal method for universal use in determining MRD status, and (c) how do the results of this test impact treatment approaches?

Several studies have demonstrated a consistent improvement in progression-free survival (PFS) with attainment of CR, but its impact on overall survival (OS) has been varied. ${ }^{12,13}$ The impact of depth of response on PFS is not surprising, as that could be a mere reflection of the time taken for the clone to grow to a measurable level following a more effective eradication. Given the toxicity and the impact on the quality of life from the intense therapies required for a $C R$, it is imperative that MRD negativity translates into improved OS, an essential step on the way to a real cure. To date two large studies have shown precisely this., ${ }^{8,10}$ Both studies have depended on flow cytometry-based detection of tumor cells to define the MRD, but other studies have used ASO-PCR-based approaches for MRD detection. In the current issue of Leukemia, Puig et al. ${ }^{9}$ present a comparative study examining the two techniques side by side. The current results, similar to a previous study, clearly demonstrate equivalent ability of the two techniques to detect residual disease, but crucial differences in terms of feasibility. ${ }^{11}$ Two issues remain critical; the inability to obtain successful primers in up to a third of the patients, and the absolute requirement for a baseline sample hampers universal adaptation of this technique. These, along with the required expertise and the more universal access to flow cytometry in most of hematology laboratories, makes flow cytometry the method of choice today, and increasing ability to interrogate millions of cells in a short time and increasing number of markers that can be accommodated at a time will lead to increasing sensitivity, specificity and ease of use of this technique. ${ }^{8,10}$ However, there is still a lack of uniformity in the cell processing methodology, specific markers required, the minimum number of cells to be studied and gating approaches that have hampered universal adoption of the technique. ${ }^{14}$ The European Myeloma Network has developed a consensus on the approach, which has been utilized in the recent MRC study and defines an aberrant phenotype as a lack of CD19 expression, strong CD56 expression, weak CD27 expression and/or weak CD45 expression. ${ }^{15}$ In patients with few events, additional markers such as CD81, CD117, CD200 and/or CD52 can be useful. ${ }^{8}$ We have found the use of light chain restriction as an important adjunct to the use of the above set of markers. ${ }^{16}$

Another missing piece in this puzzle is what to do with the information regarding MRD status. There is no doubt attainment of MRD negativity is a desirable end point, but it reflects disease biology as much as it does the treatment approach utilized. So, for a given patient, does lack of MRD negativity after a particular treatment approach call for more therapy or if MRD negativity is achieved, does it suggest the need for no additional therapy? Given that additional and often more intense therapy is likely to result in toxicities and affect the quality of life, are there particular patients in whom MRD status is not that important and hence can be spared the additional burden of therapy? Studies in the context of CR status suggest that achievement of $C R$ is critical only in patients with more aggressive or high-risk disease, while those with more indolent biology may not particularly benefit. The recent MRC study of MRD also appears to substantiate this. ${ }^{8}$ So there is clearly a tipping point where the cost of therapy (financial and quality of life) justifies the goal of achieving MRD negativity, and is likely determined by the disease biology. This question is likely to require prospectively designed studies utilizing responseadapted therapy strategies that also incorporates biological risk stratification methods.

The MRD debate in myeloma also highlights certain unique aspects of this disease compared to the other hematological disease where MRD has become an accepted clinical practice. All patients with myeloma have a preexisting stage of monoclonal gammopathy of undetermined significance (MGUS), characterized by clonal plasma cells that have not undergone the 'malignant switch' that characterizes the progression to active myeloma. ${ }^{17}$ In a situation analogous to high-grade lymphoma arising in the background of follicular low-grade lymphoma, it is possible that an eradication of the malignant clone can be achieved, leaving behind a clonal 'MGUS' population, but with excellent outcomes. However, the current immunophenotypic approaches may not be able to distinguish between the two clones without additional molecular markers of malignant switch. Whole-genome sequencing based approaches that are being studied may allow better discrimination between these states. Another aspect of MRD that requires highlighting is that the flow cytometry-based assessment of MRD is likely to be complimentary to current conventional methods of monoclonal protein assessment, as myeloma cells can survive outside of the marrow, which is the subject of MRD assessment. This is clearly highlighted by the different outcomes seen among MRD-negative patients with and without a $\mathrm{CR}^{8}{ }^{8}$ In future, sensitive imaging techniques may also compliment this assessment of a true tumor eradication 
(a.k.a cure), which will allow assessment of all potential tumor compartments. ${ }^{18}$ Finally, MRD assessments can be combined with conventional risk factors to predict risk of treatment failure as shown by Paiva et al., ${ }^{19}$ where high-risk FISH and MRD positivity were the only predictors of un-sustained $C R$ after autologous transplant.

\section{CONFLICT OF INTEREST}

The authors declare no conflict of interest.

SK Kumar and SV Rajkumar Division of Hematology, Mayo Clinic, Rochester, MN, USA E-mail: rajkumar.vincent@mayo.edu

\section{REFERENCES}

1 Barlogie B, Crowley J. Could CR mean cure? Blood 2011; 118: 483.

2 Rajkumar SV. Treatment of myeloma: cure vs control. Mayo Clin Proc 2008; 83: 1142-1145

3 Hari P, Pasquini MC, Vesole DH. Cure of multiple myeloma-more hype, less reality. Bone Marrow Transplant 2006; 37: 1-18.

4 Emig M, Saussele S, Wittor H, Weisser A, Reiter A, Willer A et al. Accurate and rapid analysis of residual disease in patients with CML using specific fluorescent hybridization probes for real time quantitative RT-PCR. Leukemia 1999; 13 1825-1832.

5 Kumar SK, Flinn I, Noga SJ, Hari P, Rifkin R, Callander N et al. Bortezomib, dexamethasone, cyclophosphamide and lenalidomide combination for newly diagnosed multiple myeloma: phase 1 results from the multicenter EVOLUTION study. Leukemia 2010; 24: 1350-1356.

6 Jakubowiak AJ, Dytfeld D, Griffith KA, Lebovic D, Vesole DH, Jagannath S et al. A phase $1 / 2$ study of carfilzomib in combination with lenalidomide and low-dose dexamethasone as a frontline treatment for multiple myeloma. Blood 2012; 120 1801-1809.

7 Cavo M, Tacchetti P, Patriarca F, Petrucci MT, Pantani L, Galli M et al. Bortezomib with thalidomide plus dexamethasone compared with thalidomide plus dexamethasone as induction therapy before, and consolidation therapy after double autologous stem-cell transplantation in newly diagnosed multiple myeloma: a randomised phase 3 study. Lancet 2010; 376: 2075-2085.

8 Rawstron AC, Child JA, de Tute RM, Davies FE, Gregory WM, Bell SE et al. Minimal residual disease assessed by multiparameter flow cytometry in multiple myeloma: impact on outcome in the Medical Research Council Myeloma IX Study. J Clin Oncol 2013; 31: 2540-2547.

9 Puig N, Sarasquete ME, Balanzategui A, Martinez J, Paiva B, Garcia H et al. Critical evaluation of ASO RQ-PCR for minimal residual disease evaluation in multiple myeloma. A comparative analysis with flow cytometry. Leukemia 2014; 28: 391-397.

10 Paiva B, Vidriales MB, Cervero J, Mateo G, Perez JJ, Montalban MA et al. Multiparameter flow cytometric remission is the most relevant prognostic factor for multiple myeloma patients who undergo autologous stem cell transplantation. Blood 2008; 112: 4017-4023.

11 Martinez-Sanchez P, Montejano L, Sarasquete ME, Garcia-Sanz R, Fernandez-Redondo E, Ayala $\mathrm{R}$ et al. Evaluation of minimal residual disease in multiple myeloma patients by fluorescent-polymerase chain reaction: the prognostic impact of achieving molecular response. $\mathrm{Br} J$ Haematol 2008; 142: 766-774.

12 Gay F, Larocca A, Wijermans P, Cavallo F, Rossi D, Schaafsma R et al. Complete response correlates with long-term progression-free and overall survival in elderly myeloma treated with novel agents: analysis of 1175 patients. Blood 2011; 117: 3025-3031

13 Harousseau JL, Palumbo A, Richardson PG, Schlag R, Dimopoulos MA, Shpilberg O et al. Superior outcomes associated with complete response in newly diagnosed multiple myeloma patients treated with nonintensive therapy: analysis of the phase 3 VISTA study of bortezomib plus melphalan-prednisone versus melphalanprednisone. Blood 2010; 116: 3743-3750.

14 Flanders A, Stetler-Stevenson M, Landgren O. Minimal residual disease testing in multiple myeloma by flow cytometry: major heterogeneity. Blood 2013; 122 1088-1089.

15 Rawstron AC, Orfao A, Beksac M, Bezdickova L, Brooimans RA, Bumbea $\mathrm{H}$ et al Report of the European Myeloma Network on multiparametric flow cytometry in multiple myeloma and related disorders. Haematologica 2008; 93: 431-438.

16 Morice WG, Hanson CA, Kumar S, Frederick LA, Lesnick CE, Greipp PR. Nove multi-parameter flow cytometry sensitively detects phenotypically distinct plasma cell subsets in plasma cell proliferative disorders. Leukemia 2007; 21: 2043-2046.

17 Landgren O, Kyle RA, Pfeiffer RM, Katzmann JA, Caporaso NE, Hayes RB et al. Monoclonal gammopathy of undetermined significance (MGUS) consistently precedes multiple myeloma: a prospective study. Blood 2009; 113: 5412-5417.

18 Bartel TB, Haessler J, Brown TL, Shaughnessy Jr. JD, van Rhee F, Anaissie E et al. F18-fluorodeoxyglucose positron emission tomography in the context of other imaging techniques and prognostic factors in multiple myeloma. Blood 2009; 114 2068-2076.

19 Paiva B, Gutierrez NC, Rosinol L, Vidriales MB, Montalban MA, Martinez-Lopez J et al. High-risk cytogenetics and persistent minimal residual disease by multiparameter flow cytometry predict unsustained complete response after autologous stem cell transplantation in multiple myeloma. Blood 2012; 119: 687-691. 